\title{
Validação de instrumento para avaliação da habilidade de mensuração da pressão arterial
}

\author{
Validation of an instrument for assessing the ability of blood pressure measurement \\ Validación de instrumento para evaluación de la habilidad de medición de la presión arterial
}

\section{Manuela Pinto Tibúrcio', Gabriela de Sousa Martins Melo', Lívia Sêmele Câmara Balduíno', Isabelle Katherinne Fernandes Costa', Thalyne Yurí de Araújo Farias Dias', Gilson de Vasconcelos Torres'}

\author{
' Universidade Federal do Rio Grande do Norte, Centro de Ciências da Saúde, Departamento de Enfermagem, \\ Programa de Pós-Graduação em Enfermagem. Natal-RN, Brasil.
}

Submissão: 25-12-2012 Aprovação: 27-06-2014

\section{RESUMO}

O estudo objetivou validar um instrumento para avaliação da habilidade dos graduandos de enfermagem para mensuração da pressão arterial. Estudo metodológico, desenvolvido com 27 enfermeiros juízes do estudo, docentes disciplina de semiologia e/ ou semiotécnica, com no mínimo 1 ano de experiência nas disciplinas. Foi realizado em duas etapas: levantamento da literatura para a elaboração do instrumento do tipo checklist e posterior submissão para validação de conteúdo mediante aplicação do Índice Kappa, aceitando-se o valor > 0,61 (nível bom), e Índice de Validade de Conteúdo (IVC) > 0,75. Com base na revisão incluíram-se 28 itens no checklist, dos quais todos obtiveram índice Kappa e IVC aceitos para a validade de conteúdo, e nove deles necessitaram de alterações. O instrumento pode ser considerado válido em seu conteúdo, com todos os itens considerados adequados, tanto separadamente, como de maneira global.

Descritores: Enfermagem; Determinação da Pressão Arterial; Estudos de Validação; Educação.

\section{ABSTRACT}

This study aimed to validate an instrument for evaluation the ability of nursing students to measure the blood pressure. It is a methodological study, developed with 27 nurses, teachers of semiology and/or semiotic disciplines, with at least 1 year of experience in disciplines, which acted as judges of the study. It was accomplished in two steps: first, a review of the literature to build the checklist instrument and subsequent submission for content validation by applying the Kappa, accepting the value > 0.61 (good level); and second, it was accomplished a Content Validity Index (CVI) $>0.75$. Based on the literature review, 28 items were included in the checklist, with all of them showing Kappa index and CVI accepted for content validity, and nine of those items needed changes. The instrument can be considered valid in its content, with all its items being considered appropriate separately, as well as globally.

Key words: Nursing; Blood Pressure Determination; Validation Studies; Education.

\section{RESUMEN}

El estudio tiene como objetivo validar un instrumento para evaluar la capacidad de los estudiantes de enfermería sobre la medición de la presión arterial. Estudio metodológico, desarrollado con 27 enfermeros jueces del estudio, profesores de la disciplina semiología y/o semiótica, con al menos 1 año de experiencia en las disciplinas. Se realizó en dos pasos: estudio de la literatura para la construcción del instrumento checklist y posterior presentación para validación de contenido mediante la aplicación del índice Kappa, aceptando el valor > 0,61 (buen nivel), y el Índice de Validez de Contenido (IVC) > 0,75. Basado en la revisión se incluyeron 28 ítems en el checklist, con todos teniendo índice de evaluación Kappa y IVC dentro de los parámetros establecidos para la validez de contenido, y nove requirieran cambios. El instrumento puede ser considerado válido en su contenido, con todos los elementos apropiados considerados tanto separados, así como en el todo.

Palabras clave: Enfermería; Determinación de la Presión Sanguínea; Estudios de Validación; Educación. 


\section{INTRODUÇÃO}

A hipertensão arterial sistêmica (HAS) é uma doença de alta prevalência, com natureza multifatorial e, muitas vezes, cursa de forma assintomática. Configura-se como o principal fator de risco cardiovascular, de morbidade e mortalidade, de forma que seu diagnóstico e controle precoce são enfatizados como importante estratégia de saúde pública ${ }^{(1-2)}$.

A medida precisa da pressão arterial (PA) é condição essencial para o diagnóstico da hipertensão, pois a sua elevação é, normalmente, o primeiro sinal da doença. Dentre os diversos métodos existentes para se determinar a PA, o método indireto com técnica auscultatória é o procedimento mais utilizado, considerado também como parâmetro que evidencia o controle ou não da hipertensão arterial. Portanto, a realização correta e precisa do procedimento é imprescindível nos programas de prevenção e tratamento de doenças cardiovasculares ${ }^{(3)}$.

Além de ser considerada padrão ouro no diagnóstico e tratamento da HAS, a medida da pressão arterial configura-se como um padrão fisiológico indispensável em toda avaliação diagnóstica, sendo um dos procedimentos mais realizados no cotidiano das instituições de saúde por qualquer membro da equipe multiprofissional, principalmente pela Enfermagem, seja em situações de emergência ou em condições ambulatoriais( ${ }^{(4)}$. É imprescindível que os profissionais possam detectar valores de PA com precisão e interpretá-los com segurança $a^{(5)}$. Apesar disso, a técnica auscultatória de pressão arterial é considerada simples e de fácil execução, e assim, muitos acabam banalizando o procedimento a ponto de torná-lo um autêntico ritual, cumprido cegamente, de maneira desleixada e desinteressada ${ }^{(6)}$.

A padronização da medida pressão arterial é uma preocupação antiga, pois, desde 1939, a American Heart Association levanta discussões sobre o procedimento e lança novas recomendações e diretrizes sempre que avanços na área são descobertos $^{(7)}$. Apesar da existência de protocolos, a mensuração da PA ainda é realizada de maneira não padronizada. A abordagem sistemática dos passos do procedimento mediante um roteiro estruturado validado, do tipo checklist, constitui-se em uma importante estratégia para auxiliar os profissionais de saúde e, mais que isso, contribuir na formação de futuros enfermeiros ${ }^{(8)}$.

Ensinar aos alunos de Graduação em Enfermagem, mantê-los atualizados e, principalmente, avaliá-los tem sido um grande desafio ${ }^{(8)}$. Diante da importância da mensuração da PA na prática da Enfermagem, é de suma importância avaliar a habilidade técnica referente a este procedimento.

O conceito de habilidade está associado ao saber fazer, ou seja, à capacidade de aplicar e fazer uso produtivo do conhecimento adquirido com vistas à consecução de um propósito específico ${ }^{(9)}$. A impossibilidade de não se poder avaliar adequadamente a habilidade dos estudantes de graduação, futuros enfermeiros, em relação ao procedimento de mensuração da PA pode culminar no comprometimento dos valores obtidos, interferindo na avaliação clínica e causando possíveis danos aos pacientes. Além disso, a avaliação torna-se importante para que eles conheçam suas fragilidades teóricas e práticas e que, assim, busquem capacitação para o exercício mais competente de sua profissão $0^{(8,10)}$.
Toda avaliação de aprendizagem requer a adequada forma de averiguar e medir a evolução de quem está sendo avaliado, do seu desempenho, além de indicar os reajustes necessários $^{(11-12)}$. Para tanto, mostra-se necessário o desenvolvimento de instrumentos de avaliação respaldados em conhecimentos científicos existentes ${ }^{(13)}$.

O reconhecimento da qualidade dos instrumentos é aspecto fundamental para a sua legitimidade e confiabilidade, o que reforça a importância do processo de validação de conteúdo, o qual é composto por diversas etapas que visam coletar evidências de que o instrumento realmente mede a variável a que se propõe e de que é útil para a finalidade proposta ${ }^{(13)}$. Duas etapas principais na validação de conteúdo são: o desenvolvimento do instrumento, que abrange as fases de identificação do fenômeno estudado, a produção dos itens do instrumento e a sua construção; e o julgamento do instrumento realizado por juízes qualificados ${ }^{(14)}$.

Tendo em vista os problemas concernentes à medida da PA, as lacunas de instrumentos para verificação da habilidade prática dos estudantes e profissionais, bem como a preocupação com a padronização da medida, o estudo teve como objetivo validar um instrumento para avaliação da habilidade dos graduandos de enfermagem para mensuração da pressão arterial.

\section{MÉTODO}

Estudo metodológico, desenvolvido no período de junho a setembro de 2012, tendo como foco a validação de conteúdo do instrumento sobre a habilidade dos graduandos de enfermagem para mensuração da pressão arterial. A validade de conteúdo se refere à análise dos itens que compõem o instrumento por juízes no assunto, sendo a determinação de representatividade e extensão com que cada item da medida comprova o fenômeno de interesse e a sua dimensão dentro daquilo que se propõe investigar ${ }^{(15)}$.

A pesquisa foi realizada em instituições de ensino de enfermagem, de nível técnico e superior no Rio Grande do Norte, envolvendo docentes da Graduação e de Nível Técnico em Enfermagem.

O estudo foi desenvolvido em duas etapas. A primeira envolveu a revisão da literatura para elaboração do instrumento. Com base nessa etapa, foi desenvolvido um roteiro de observação estruturado, do tipo checklist, composto por 28 itens divididos em três blocos (observações iniciais, mensuração da pressão arterial e observações finais), que correspondem aos passos considerados importantes para se avaliar a habilidade técnica para mensuração da pressão arterial.

Na segunda etapa, ocorreu a identificação e seleção da população alvo a ser recrutada para participar como juízes, mediante um contato inicial com as coordenações dos Cursos de Enfermagem de Universidades do Rio Grande do Norte. Apresentaram-se a eles os critérios de inclusão para seleção dos participantes: atuação na disciplina de semiologia e/ou semiotécnica há pelo menos um ano. Foram identificados 34 docentes elegíveis, para os quais foi enviada uma carta convite, por via eletrônica, contendo os objetivos e a justificativa do estudo.

Dos 34 docentes convidados, 28 aceitaram participar. $\mathrm{O}$ instrumento, um roteiro do processo de avaliação do 
instrumento e o termo de consentimento livre e esclarecido foram entregues pessoalmente a cada um destes. A avaliação de um juiz estava incompleta, e assim foi considerada como uma perda, de modo que a população alvo do estudo foi constituída por 27 juízes.

Os instrumentos foram avaliados por item e de forma global, considerando 10 requisitos: utilidade/pertinência, consistência, clareza, objetividade, simplicidade, exequibilidade, atualização, vocabulário, precisão, sequência instrucional de tópicos, devendo os juízes julgarem se o requisito estava adequado, adequado com alterações ou inadequado. Os juízes também deveriam designar, de forma escrita, sugestões a fim de que os itens pudessem ser melhorados.

Após a avaliação, foi realizada a validação de conteúdo com aplicação do Índice Kappa (K) para mensuração do nível de concordância e consistência dos juízes em relação à permanência ou não dos itens do instrumento, levando em conta as indicações de "inadequado" para os mesmos. O Índice Kappa é um indicador de concordância ajustado que varia de "menos 1" a "mais 1" - quanto mais próximo de 1 melhor o nível de concordância entre os observadores; sua distribuição e os respectivos níveis de interpretação são: $<0,00=$ ruim; 0,00 a $0,20=$ fraco; 0,21 a $0,40=$ sofrível; 0,41 a $0,60=$ regular; 0,61 a $0,80=$ bom; 0,81 a 0,99= ótimo; $1,00=$ perfeito ${ }^{(16)}$. Como critério de aceitação, foi estabelecida a concordância superior a 0,61 entre os juízes.

Também foi utilizado o Índice de Validade de Conteúdo (IVC), que mede a concordância dos juízes quanto à representatividade dos itens em relação ao conteúdo em estudo ${ }^{(15)}$, sendo calculado dividindo-se o número de juízes que avaliaram o item como adequado/adequado necessitando de alterações pelo total de juízes (avaliação por item), resultando na proporção de juízes que julgaram o item válido ${ }^{(17)}$.

Para calcular o IVC geral do instrumento foi realizada a soma de todos os IVC calculados separadamente, dividido pelo número de itens ${ }^{(17)}$. Como aceitável, considerou-se índice mínimo de 0,75 tanto para avaliação de cada item como para avaliação geral do instrumento.

Os dados foram organizados em planilha de dados eletrônica e exportados para um software estatístico. Depois de codificados e tabulados, foram analisados por meio de estatística descritiva. Os instrumentos foram reformulados de acordo com as sugestões dos juízes.

O estudo obteve parecer favorável do Comitê de Ética em Pesquisa/HUOL, Certificado de Apresentação para Apreciação Ética (CAAE) nº 0002.0.294.000-10.

\section{RESULTADOS}

Com relação aos juízes, 74,1\% apresentavam como máxima titulação o mestrado acadêmico e $63 \%$ exerciam a docência exclusivamente. A idade variou de no mínimo de 25 anos e máximo de 57, média de 36,6 ( \pm 9,0) anos. O tempo de experiência na docência variou entre 1 a 34 anos, com média de 7,9 $( \pm 8,0)$ anos. Quanto ao tempo de experiência nas disciplinas de semiologia e/ou semiotécnica da Enfermagem, variou entre 1 e 32 anos, com média de 5,5 $( \pm 6,7)$ anos.

Em se tratando do processo de julgamento dos itens que compõem o checklist da técnica de mensuração da pressão arterial, nenhum deles foi avaliado como inadequado. Todos obtiveram concordância dentro do nível estabelecido (IVC > 0,75 e Kappa > 0,61). Os resultados são apresentados na Tabela 1.

Tabela 1 - Julgamento dos juízes $(n=27)$ sobre itens do checklist da habilidade para avaliação da técnica de mensuração da pressão arterial. Natal-RN, 2012

JULGAMENTO

Itens referentes à habilidade para a técnica de mensuração da pressão arterial

\begin{tabular}{cccc}
\multicolumn{4}{c}{ JULGAMENTO } \\
\hline Adequado & $\begin{array}{c}\text { Adequado c/ } \\
\text { alterações }\end{array}$ \\
\cline { 1 - 2 } IVC & $\%$ & $\mathrm{n}$ & $\%$
\end{tabular}

\section{OBSERVAÇÕES INICIAIS}

1.1 Verifica dados no prontuário do usuário

1.2 Higieniza as mãos

1.3 Seleciona o material e os instrumentos necessários

1.4 Identifica-se para o paciente e explica o procedimento a ser realizado

$\begin{array}{rrrrrr}26 & 96,3 & 1 & 3,7 & 0,96 & 0,93 \\ 27 & 100,0 & - & - & 1,00 & 1,00 \\ 25 & 92,6 & 2 & 7,4 & 0,93 & 0,86 \\ 27 & 100,0 & - & - & 1,00 & 1,00\end{array}$

\section{MENSURAÇÃO DA PRESSÃO ARTERIAL}

2.1 Investiga possíveis condições fisiológicas e externas que possam alterar a mensuração da pressão arterial

2.2 Mantém o usuário em repouso

2.3 Mede a circunferência do braço do cliente

2.4 Verifica a posição do cliente

2.5 Seleciona o manguito adequado

2.6 Localiza artéria braquial por palpação

2.7 Ajusta o manguito selecionado no braço

$\begin{array}{rrrrrr}24 & 88,9 & 3 & 11,1 & 0,89 & 0,79 \\ 25 & 92,6 & 2 & 7,4 & 0,93 & 0,86 \\ 24 & 88,9 & 3 & 11,1 & 0,89 & 0,79 \\ 24 & 88,9 & 3 & 11,1 & 0,89 & 0,79 \\ 25 & 92,6 & 2 & 7,4 & 0,93 & 0,86 \\ 25 & 92,6 & 2 & 7,4 & 0,93 & 0,86 \\ 26 & 96,3 & 1 & 3,7 & 0,96 & 0,93\end{array}$


2.8 Mantém o braço do usuário bem apoiado na altura do coração

2.9 Posiciona os olhos no mesmo nível da coluna de mercúrio ou do manômetro aneroide

2.10 Estima o nível da pressão sistólica

2.11 Desinfla o manguito rapidamente e aguarda antes de iniciar a medida

2.12 Faz a desinfecção da campânula e das olivas do estetoscópio

2.13 Posiciona o estetoscópio no ouvido

2.14 Posiciona a campânula do estetoscópio sobre a artéria braquial

2.15 Infla rapidamente o manguito

2.16 Procede à deflação lentamente

2.17 Determina a pressão sistólica

2.18 Determina a pressão diastólica

2.19 Procede à deflação do manguito

2.20 Realiza novas medidas, se necessário

\section{OBSERVAÇÕES FINAIS}

3.1 Informa ao usuário o valor da pressão arterial verificado

3.2 Organiza o ambiente

3.3 Higieniza as mãos

3.4 Registra o procedimento no prontuário

$\begin{array}{rrrrrr}27 & 100,0 & - & - & 1,00 & 1,00 \\ 26 & 96,3 & 1 & 3,7 & 0,96 & 0,93 \\ 23 & 85,2 & 4 & 14,8 & 0,85 & 0,74 \\ 25 & 92,6 & 2 & 7,4 & 0,93 & 0,86 \\ 24 & 88,9 & 3 & 11,1 & 0,89 & 0,79 \\ 27 & 100,0 & - & - & 1,00 & 1,00 \\ 27 & 100,0 & - & - & 1,00 & 1,00 \\ 24 & 88,9 & 3 & 11,1 & 0,89 & 0,79 \\ 26 & 96,3 & 1 & 3,7 & 0,96 & 0,93 \\ 23 & 85,2 & 4 & 14,8 & 0,85 & 0,74 \\ 23 & 85,2 & 4 & 14,8 & 0,85 & 0,74 \\ 27 & 100,0 & - & - & 1,00 & 1,00 \\ 24 & 88,9 & 3 & 11,1 & 0,89 & 0,79\end{array}$

$\begin{array}{llllll}25 & 92,6 & 2 & 7,4 & 0,93 & 0,86\end{array}$

$27100,0 \quad-\quad-1,00 \quad 1,00$

$27100,0 \quad-\quad-1,001,00$

$27100,0 \quad-\quad-1,001,00$
Conforme Tabela 1, do total de 28 itens no checklist, 9 apresentaram índice de concordância perfeito (IVC=1,00; Kappa = 1,00).

No bloco da mensuração da pressão arterial, os itens onde houve maior número de opinião de que eram considerados adequados, mas exigiam alterações foram: investiga possíveis condições fisiológicas e externas que possam alterar a mensuração da pressão arterial (item 2.1); mede a circunferência do braço do cliente (item 2.3); verifica a posição do cliente (item 2.4); estima o nível da pressão sistólica (item 2.10); faz a desinfecção da campânula e das olivas do estetoscópio (item 2.12); infla rapidamente o manguito (item 2.15); determina a pressão sistólica (item 2.17); determina a pressão diastólica (item 2.18); realiza novas medidas, se necessário (item 2.20).

No Quadro 1, são apresentados os itens avaliados como "adequado com alterações", os requisitos relacionados ao problema e as sugestões para melhoria ou reformulação dos itens.

Quadro 1 - Sugestões dos juízes acerca dos itens considerados adequados com alterações para avaliação da habilidade para mensuração da pressão arterial. Natal-RN, 2012

\begin{tabular}{|l|l|l|}
\hline \multicolumn{1}{|c|}{ Itens } & Requisitos avaliados que exigiram alterações & \multicolumn{1}{c|}{ Sugestões dos juízes } \\
\hline $\begin{array}{l}\text { Investiga possíveis condições } \\
\text { fisiológicas e externas que possam } \\
\text { alterar a mensuração da pressão arterial }\end{array}$ & $\begin{array}{l}\text { Clareza; } \\
\text { Sequencia instrucional dos tópicos. }\end{array}$ & $\begin{array}{l}\text { * Detalhar no instrumento as condições fisiológicas e } \\
\text { externas que possam alterar a mensuração da pressão arterial. } \\
\text { * Colocar o item no bloco das observações iniciais. }\end{array}$ \\
\hline $\begin{array}{l}\text { Mede a circunferência do braço do } \\
\text { cliente }\end{array}$ & $\begin{array}{l}\text { Consistência; Exequível; Sequência } \\
\text { instrucional dos tópicos. }\end{array}$ & $\begin{array}{l}\text { *Descrever mais detalhadamente como essa medição deve } \\
\text { ocorrer. } \\
\text { * Etapa difícil de ser realizada nos serviços. } \\
\text { * item deve ser colocado após o 2.4. }\end{array}$ \\
\hline Verifica a posição do cliente & Clareza; Vocabulário & $\begin{array}{l}\text { * Detalhar o posicionamento adequado do cliente. } \\
\text { * Reformular o item para: posiciona o cliente. }\end{array}$ \\
\hline Estima o nível da pressão sistólica & Clareza & $\begin{array}{l}\text { * Descrever detalhadamente a etapa, utilizando o termo } \\
\text { método palpatório. }\end{array}$ \\
\cline { 1 - 2 } $\begin{array}{l}\text { Faz a desinfecção da campânula e } \\
\text { das olivas do estetoscópio }\end{array}$ & Sequência instrucional dos tópicos & $\begin{array}{l}\text { * O item deve ser inserido no bloco das observações } \\
\text { iniciais. }\end{array}$ \\
\hline Infla rapidamente o manguito & Clareza & * Descrever detalhadamente a etapa. \\
\hline Determina a pressão sistólica/ & Vocabulário & * Trocar o verbo “determina" por "identifica". \\
\hline Determina a pressão diastólica & Atualização & $\begin{array}{l}\text { * Rever o intervalo de tempo entre as medidas na literatura } \\
\text { mais atual. }\end{array}$ \\
\hline Realiza novas medidas, se necessário
\end{tabular}


No parecer final dos juízes acerca do checklist, baseado nos 10 requisitos de avaliação, todos os requisitos obtiveram índice Kappa bom e IVC > 0,75, como mostra a Tabela 2. Desses, utilidade/pertinência e simplicidade receberam escore máximo de concordâncias. Em contrapartida, os itens clareza, sequência instrucional dos tópicos e vocabulário mereceram atenção especial, sendo incorporadas as sugestões e observações dos juízes para a versão final do instrumento.

Tabela 2 - Parecer final dos juízes $(n=27)$ acerca do checklist de avaliação das habilidades procedimentais da técnica de mensuração da pressão arterial. Natal-RN, 2012

\begin{tabular}{|c|c|c|c|c|c|c|}
\hline \multirow{3}{*}{ REQUISITOS } & \multicolumn{6}{|c|}{ PARECER FINAL CHECK LIST } \\
\hline & \multicolumn{2}{|c|}{ Adequado } & \multicolumn{2}{|c|}{$\begin{array}{c}\text { Adequado c/ } \\
\text { alterações }\end{array}$} & \multirow[t]{2}{*}{ IVC } & \multirow[t]{2}{*}{$\mathrm{K}$} \\
\hline & $\mathrm{n}$ & $\%$ & $\mathrm{n}$ & $\%$ & & \\
\hline Utilidade/ pertinência & 27 & 100,0 & - & - & 1,00 & 1,00 \\
\hline Simplicidade & 27 & 100,0 & - & - & 1,00 & 1,00 \\
\hline Exequível & 26 & 96,3 & 1 & 3,7 & 0,96 & 0,93 \\
\hline Objetividade & 26 & 96,3 & 1 & 3,7 & 0,96 & 0,93 \\
\hline Atualização & 26 & 96,3 & 1 & 3,7 & 0,96 & 0,93 \\
\hline Precisão & 26 & 96,3 & 1 & 3,7 & 0,96 & 0,93 \\
\hline Consistência & 25 & 92,6 & 2 & 7,4 & 0,93 & 0,86 \\
\hline Clareza & 24 & 88,9 & 3 & 11,1 & 0,89 & 0,79 \\
\hline Sequência instrucional dos tópicos & 24 & 88,9 & 3 & 11,1 & 0,89 & 0,79 \\
\hline Vocabulário & 23 & 85,2 & 4 & 14,8 & 0,85 & 0,74 \\
\hline
\end{tabular}

procedimento ao paciente foi a etapa mais realizada $(97,1 \%)$ (3). Por mais que o contato inicial profissional/cliente não interfira diretamente valores da mensuração da pressão arterial, o ato do profissional se identificar ao cliente, explicitando seu nome e sua função, bem como o procedimento a ser executado, de forma clara e simples, é imprescindível para o esclarecimento de dúvidas e, consequentemente, para a redução do medo e da ansiedade.

Essa medida toma proporções ainda maiores pela constatação de que o estado emocional do cliente está diretamente relacionado a dois fenômenos conhecidos como reação de alarme e hipertensão do jaleco branco, que levam ao aumento dos níveis tensionais devido a resposta do cliente frente ao profissional de saúde, principalmente o médico, que ainda Ihe é pessoa estranha, ou mesmo devido à ansiedade gerada pela expectativa de algum desfecho desconhecido e doloroso ${ }^{(6)}$.

No bloco da mensuração da pressão arterial, os juízes sugeriram alterações em alguns itens, entre eles "mede a circunferência do braço do cliente" e "verifica a posição do cliente". Com relação ao primeiro item, alguns juízes avaliaram-no como não exequível, uma vez que manguitos de diferentes tamanhos não são encontrados habitualmente nos serviços de saúde. O uso do manguito com tamanho inadequado em relação à circunferência do braço $(C B)$ é uma das causas mais discutidas de medida imprecisa da PA, podendo fazer com que ela seja super ou subestimada. A razão da circunferência braquial/largura do manguito deve ser em torno de 0,40 e o comprimen-

Na avaliação global, o checklist para avaliação das habilidades procedimentais da técnica, de mensuração da pressão arterial obteve IVC de 0,94 e Kappa de 0,89.

\section{DISCUSSÃO}

Apesar dos profissionais de enfermagem serem os mais habituados com a mensuração da pressão arterial ${ }^{(3)}$, estudos evidenciam a realização do procedimento de maneira incorreta, com erros ou omissão de cuidados básicos durante a aferição ${ }^{(3,18)} \mathrm{e}$ lacunas no conhecimento teórico-prático, sugerindo avaliação prática da execução do procedimento e elaboração de estratégias específicas de acompanhamento dos enfermeiros, a fim de evitar defasagem do seu conhecimento sobre o assunto ${ }^{(19)}$.

A higienização das mãos, seja ela com água e sabão ou com soluções alcoólicas, é prática reconhecida e recomendada no âmbito dos serviços de saúde, devendo ser realizada corretamente antes e após todo e qualquer procedimento, incluindo a mensuração da pressão arterial. Apesar de todas as evidências mostrarem a importância das mãos na cadeia de transmissão das infecções hospitalares, observa-se, na prática, a baixa adesão a essa técnica por parte dos profissionais ou, quando ela é realizada, habitualmente é desenvolvida de forma incorreta, principalmente em relação a sua duração(18).

Em um estudo com o objetivo de avaliar como a medida da pressão arterial é realizada por profissionais, a explicação do to do manguito deve circundar de 80 a $100 \%$ da $\mathrm{CB}^{(7)}$.

Pesquisa mostra que 78,0\% dos profissionais de várias categorias referiram não dispor de manguitos de diferentes dimensões no seu ambiente de trabalho. Com isso, ao serem avaliados durante o procedimento de mensuração da PA, a etapa de seleção da largura do manguito adequada foi realizada por apenas $6,7 \%$ deles $^{(3)}$. Outros estudiosos da área questionam se os profissionais da saúde seriam capazes de selecionar tamanhos apropriados dos manguitos, mesmo se esses fossem disponibilizados nas unidades médicas. Eles também constataram que apenas o manguito padrão era disponibilizado nas enfermarias e que o pessoal responsável pela compra desse equipamento não havia recebido orientações acerca da possibilidade de adquirir outros tamanhos de manguitos já disponíveis no mercado(20).

Quanto ao item "verifica a posição do cliente", além da alteração do vocabulário foi sugerido que o posicionamento correto do cliente fosse mais bem descrito. Durante a mensuração da PA, o cliente deve estar em posição sentada, com dorso recostado na cadeira, pernas descruzadas e pés apoiados no chão, o braço deve estar situado na altura do coração, descoberto, apoiado, com a palma da mão voltada para cima e cotovelo ligeiramente fletido ${ }^{(7)}$.

Em relação ao método palpatório, observa-se que, na prática, normalmente não se realiza a estimativa da pressão sistólica por meio da palpação artéria radial. Geralmente, 
o executor infla o manguito até um ponto decidido aleatoriamente por ele mesmo e, em seguida, o desinfla fazendo a ausculta dos sons de Korotkoff(21). Dessa forma, os juízes recomendaram melhorar a clareza do item "estima o nível da pressão sistólica", descrevendo detalhadamente a técnica, chamando atenção para o método palpatório.

O desconhecimento do método palpatório, em algumas ocasiões, pode levar a erros na mensuração da PA, como por exemplo, na presença do hiato auscultatório, que corresponde a um intervalo silencioso representado pela ausência da fase II da escala de Korotkoff. Ressalta-se que caso a mensuração da pressão arterial não inclua a estimativa da pressão sistólica, o observador pode, nos pacientes com hiato auscultatório, não identificar corretamente o som correspondente à pressão sistólica ou então superestimar a pressão diastólica ${ }^{(21)}$.

Em Procedimentos Operacionais Padrão e outros planos de cuidados $^{(22)}$, consta que a desinfecção da campânula e das olivas do estetoscópio deve ser realizada antes do contato com o paciente, sendo recomendada a inserção desse item no bloco das observações iniciais.

Nas etapas "infla rapidamente o manguito" e "realiza novas medidas, se necessário", os juízes recomendaram, respectivamente, descrever detalhadamente quanto à clareza e atualização, sendo especificado que o manguito deverá ser inflado rapidamente até ultrapassar 20 a 30mmHg do nível da pressão sistólica estimado no método palpatório, e que o intervalo de tempo entre as medidas na literatura é em torno de 1 minuto, como recomendam as diretrizes da Sociedade Brasileira de Cardiologia ${ }^{(7)}$.

No bloco observações finais, três, dos quatro itens, apresentaram nível de concordância perfeito. Desses, merece destaque o item "registra o procedimento no prontuário", no qual deverão ser anotados os valores da pressão sistólica e diastólica, o braço em que a PA foi medida, a posição em que o paciente se encontrava e o tamanho do manguito utilizado. Vale salientar que não é correto fazer aproximações ou arredondamentos dos números terminados em zero ou cinco ${ }^{(4,7)}$.

Pesquisa evidenciou a preferência pelo registro de valores com dígito zero para a pressão arterial sistólica em 77,1\% e para a pressão arterial diastólica em $74,3 \%$ das medidas ${ }^{(3)}$. Essa prática pode induzir a falso dado de normotensão ou de hipertensão arterial.
A mensuração da pressão arterial é um dos procedimentos mais realizados nos serviços de saúde. No entanto, durante a sua realização por meio do método indireto, se os cuidados básicos e indispensáveis em relação ao cliente, ao equipamento, à técnica e ao registro dos valores não forem obedecidos, a falta de precisão na medida da pressão arterial será inevitável e, consequentemente, julgamentos imprecisos do estado de saúde da população poderão ser estabelecidos.

Os índices obtidos no processo de validação de conteúdo do instrumento estudado indicaram alta confiabilidade e fidedignidade do mesmo para a avaliação das habilidades procedimentais na mensuração da pressão arterial pelos estudantes de enfermagem. Ele pode ser útil também como estratégia de ensino, uma vez que pode guiar claramente os estudantes quanto às etapas operacionais indicadas.

Podem ser identificadas, como limitações do estudo, a técnica de seleção dos juízes, que restringiu o seu quantitativo e distribuição geográfica.

Considera-se ainda que este instrumento deva ser continuamente aperfeiçoado e utilizado na formação e avaliação de outros profissionais. A expectativa é que o instrumento validado seja divulgado e utilizado tanto pelas instituições formadoras para avaliar seus alunos, tanto em laboratórios de habilidades ou em campos de prática clínica, como também pelas instituições de saúde, mediante programas de educação continuada com o intuito de promover atualização e melhoria na capacidade técnica de sua equipe de profissionais.

\section{CONCLUSÃO}

O checklist para avaliação da habilidade dos graduandos de enfermagem para mensuração da pressão arterial mostrou-se válido quanto ao conteúdo. Todos os itens, separadamente, e o instrumento de forma global foram avaliados como adequados para mensuração da habilidade para mensuração da pressão arterial, de acordo com os requisitos determinados.

Do total de 28 itens no checklist, nove necessitaram de alterações, principalmente no que diz respeito à clareza, sequência instrucional de tópicos e vocabulário. Na avaliação global, o instrumento obteve IVC de 0,94 e Kappa de 0,89, bem superior do considerado aceitável. As sugestões dos juízes foram acatadas, buscando o aumento da clareza dos itens, facilitando a leitura, o entendimento e aplicabilidade dos instrumentos.

\section{REFERÊNCIAS}

1. Christofaro DGD, Andrade SM, Fernandes RA, Cabrera MAS, RittiDias RM. Prevalência de pressão arterial elevada em crianças e adolescentes: revisão sistemática. Rev Bras Saúde Matern Infant [Internet]. 2011. [acesso em 25 de outubro de 2012];11(4):361-7. Disponível em: http://www.scielo.br/scielo.php?pid = S1519-382920110 00400002\&script $=$ sci_arttext

2. Oliveira SMJV, Santos JLF, Lebrão ML, Duarte YAO, Pierin AMG. Hipertensão arterial referida em mulheres idosas: prevalência e fatores associados. Texto \& Contexto Enferm [Internet]. 2008 [acesso em 25 de outubro de 2012]; 17(2):241-9. Disponível em: http://www.scielo.br/pdf/tce/v17n2/04.pdf

3. Veiga EV, Nogueira MS, Cárnio EC, Marques S, Lavrador MAS, Moraes SA, et al. Avaliação de técnicas da medida da pressão arterial pelos profissionais de saúde. Arq Bras Cardiol [Internet]. 2003 [acesso em 04 de outubro de 2012];80(1):83-9. Disponível em: http://www.scielo. br/pdf/abc/v80n1/14382.pdf 
4. Geleilete TJM, Coelho EB, Nobre F. Medida casual da pressão arterial. Rev Bras Hipertens [Internet]. 2009 [acesso em 15 de outubro 2012];16(2):118-22. Disponível em: http://departamentos.cardiol.br/dha/revista/16-2/13-medida.pdf

5. Andrade LZC, Freitas DT, Holanda GF, Silva VM, Lopes MVO, Araújo TL. Desenvolvimento e validação de jogo educativo: medida da pressão arterial. Rev Enferm UERJ [Internet]. 2012 [acesso em 26 de outubro de 2012];20(3):323-7. Disponível em: http://www.facenf. uerj.br/v20n3/v20n3a07.pdf

6. Kloetzel K. Uma lição esquecida: como ler corretamente a pressão arterial. Diagn Tratamento [Internet]. 2003 [acesso em 10 de outubro de 2012];8(4):171-82. Disponível em: http://bases.bireme.br/cgi-bin/wxislind.exe/iah/ online/? lsisScript $=$ iah/iah. $x i s \& s r c=$ google $\&$ base $=$ LILAC S\&lang $=$ p\&nextAction $=$ Ink\&exprSearch $=508037$ \&inde $\mathrm{xSearch}=\mathrm{ID}$

7. Sociedade Brasileira de Cardiologia, Sociedade Brasileira de Hipertensão, Sociedade Brasileira de Nefrologia. VI Diretrizes Brasileiras de Hipertensão. Arq Bras Cardiol [Internet]. 2010. [acesso em 17 de agosto de 2012];95(1Suppl.1):1-51. Disponível em: http://www.scielo.br/pdf/abc/ v95n1s1/v95n1s1.pdf

8. Alavarce DC, Pierin, AMG. Elaboração de uma hipermídia educacional para o ensino do procedimento de medida da pressão arterial. Rev Esc Enferm USP [Internet]. 2011. [acesso em 26 de outubro de 2012]; 45(4):939-44. Disponível em: http://www.scielo.br/pdf/reeusp/v45n4/ v45n4a21.pdf

9. Santo AP. Conhecimentos, habilidades e atitudes: o conceito de competências no trabalho e seu uso no setor público. Rev Serviço Público [Internet]. 2011 [acesso em 26 de outubro de 2012];62(4):369-86. Disponível em: http://www. enap.gov.br/index.php?option $=$ com_docman\&task $=$ doc

10. Silva SSBE, Colósimo FC, Pierin AMG. O efeito de intervenções educativas no conhecimento da equipe de enfermagem sobre hipertensão arterial. Rev Esc Enferm USP [Internet]. 2010 [acesso em 28 de maio de 2014];44(2):488-9. Disponível em: http://www.scielo.br/ $\mathrm{pdf} /$ reeusp/v44n2/35.pdf

11. Moura ECC, Mesquita LFC. Estratégias de ensino-aprendizagem na percepção de graduandos de enfermagem. Rev Bras Enferm [Internet]. 2010 [acesso em 11 de outubro de 2012];63(5):793-8. Disponível em: http://www.scielo.br/scielo.php?script=sci_arttext\&pid $=$ S0034-71672010000500016

12. Rodrigues RM, Caldeira S. Formação na graduação em enfermagem no estado do Paraná. Rev Bras Enferm [Internet]. 2009 [acesso em 12 de outubro de 2012];62(3):417-23. Disponível em: http:// www.scielo.br/scielo.php?script =sci_arttext\&pid $=$ S0034-71672009000300013

13. Bittencourt HR, Creutzberg M, Rodrigues ACM, Casartelli AO, Freitas ALS. Desenvolvimento e validação de um instrumento para avaliação de disciplinas na educação superior. Est Aval Educ [Internet]. 2011 [acesso em 15 de outubro de 2012];22(48):91-114. Disponível em: http:// educa.fcc.org.br/pdf/eae/v22n48/v22n48a06.pdf

14. Lynn MR. Detemination and quantificartion of content validity. Nurs Res [Internet]. 1986 [cited 2012 october 15];35(6):382-5. Available from: http://www.ncbi.nlm. nih.gov/pubmed/3640358

15. Rubio DM, Berg-Weger M, Tebb SS, Lee ES, Rauch S. Objectifying content validity: conducting a content validity study in social work research. Soc Work Res [Internet]. 2003 [cited 2012 august 17]; 27(2):94-104. Available from: http://swr.oxfordjournals.org/content/27/2/94.full. $\mathrm{pdf}+\mathrm{html}$

16. Pereira MG. Epidemiologia: teoria e prática. Rio de Janeiro (RJ): Guanabara Koogan; 1995.

17. Polit DF, Beck CT. The content validity index: are you sure you know what's being reported? Critique and recommendations. Res Nurs Health [Internet]. 2006 [cited 2012 october 9];29(5):489-97. Available from: http://www. ncbi.nlm.nih.gov/pubmed/16977646

18. World Health Organization. WHO guidelines on hand hygiene in health care. First global patient safety challenge clean care is safer care. Geneva: WHO; 2007.

19. Almeida TCF, Lamas JLT. Enfermeiros de Unidade de Terapia Intensiva adulto: avaliação sobre medida direta e indireta da pressão arterial. Rev Esc Enferm USP [Internet]. 2013 [acesso em 28 de maio de 2014];47(2):369-76. Disponível em: http://www.revistas.usp.br/reeusp/article/ view/58514/0

20. Veiga EV, Arcuri EAM, Cloutier L, Santos JLF. Medida da pressão arterial: circunferência braquial e disponibilidade de manguitos. Rev Latinoam Enferm [Internet]. 2009 [acesso em 06 de outubro de 2012];17(4):455-61. Disponível em: http://www.scielo.br/pdf/rlae/v17n4/pt_04.pdf

21. Ribeiro CCM, Lamas JLT. Comparação entre as técnicas de mensuração da pressão arterial em um e em dois tempos. Rev Bras Enferm [Internet]. 2012 [acesso em 28 de maio de 2014];65(4):630-6. Disponível em: http://www. scielo.br/pdf/reben/v65n4/a12v65n4.pdf

22. Tavares EO, Oliveira MLF. Padrões mínimos de atendimento inicial à urgência toxicológica para abordagem à criança intoxicada. Rev RENE [Internet]. 2012 [acesso em 28 de maio de 2014];13(1):147-57. Disponível em: http:// www.revistarene.ufc.br/revista/index.php/revista/article/ view/26/22 